\title{
BOUNDED POINT EVALUATIONS AND POLYNOMIAL APPROXIMATION
}

\author{
JAMES E. THOMSON
}

(Communicated by T. W. Gamelin)

\begin{abstract}
We consider the set of bounded point evaluations for polynomials with respect to the $L^{P}$-norm for a measure. We give an example of a measure where the corresponding sets of bounded point evaluations vary with the exponent $p$. The main ingredient is the remarkable work of $\mathrm{K}$. Seip on interpolating and sampling sequences for weighted Bergman spaces.
\end{abstract}

\section{INTRODUCTION}

For a positive measure $\mu$ with compact support in the complex plane and for $1 \leq t<\infty$ let $P^{t}(\mu)$ denote the closure in $L^{t}(\mu)$ of the analytic polynomials. A point $w$ is a bounded point evaluation (bpe) for $P^{t}(\mu)$ if there exists a constant $M$ such that $|p(w)| \leq M\|p\|$ for every polynomial $p$. In [8] we describe $P^{t}(\mu)$ and establish the existence of a large open set of bpes if $P^{t}(\mu) \neq L^{t}(\mu)$. The purpose of this paper is to give examples of measure where the corresponding sets of bpes vary with the exponent $t$.

In all previously known examples the set of bpes is independent of the exponent $t$. For example, if $\mu$ is supported on the unit circle, then Szego's theorem implies that the set of bpes is determined by point masses and the RadonNikodym derivative of $\mu$ with respect to Lebesgue measure. If the derivative is log integrable, then the set of bpes includes the open unit disk. If not, then $P^{t}(\mu)=L^{t}(\mu)$ for all $t$ and all bpes arise from point masses. However, if Lebesgue measure is absolutely continuous with respect to $\mu$, then the set

$$
\left\{f d \mu: f \in L^{1}(\mu)\right\}
$$

includes all the Poisson kernels as measures; and hence point evaluations at points in the open unit disk are weak-star continuous. (Here we are considering the polynomials as a subset of $L^{\infty}(\mu)$, which has a weak-star topology.) It follows that there exists a measure $\mu$ on the circle with no bounded point evaluations for $t<\infty$ but with weak-star continuous evaluations.

Historically, polynomial approximation with respect to area measure on simply connected regions has been studied extensively. References to major results and examples can be found in [2] and [5]. More recently, John Akeroyd [1] has

Received by the editors June 16, 1993 and, in revised form, September 27, 1993.

1991 Mathematics Subject Classification. Primary 30E05, 30E10, 46E15. 
determined the set of bpes for $P^{t}(\mu)$ for a large class of crescents, where $\mu$ is harmonic measure on the boundary of the crescent.

Our examples are based on the remarkable work of Kristian Seip [6, 7]. Seip completely describes the interpolating and sampling sequences for weighted Bergman spaces. He also gives examples of sequences that lie on the edge between the two concepts. Let $A$ denote normalized Lebesgue measure on the open unit disk $U$. Let $a$ and $b$ be positive numbers, and let $\mu$ be the measure with $d \mu=\left(1-|z|^{2}\right)^{2 a-1} d A$. Seip constructs a sequence $\Gamma$ in $U$ (depending only on $b$ ) such that $\Gamma$ is interpolating for $P^{2}(\mu)$ if $a>b$ and $\Gamma$ is a set of sampling for $P^{2}(\mu)$ if $a<b$. This example leads to our examples.

Our first example is an atomic measure $\mu$ with the property that $P^{t}(\mu)=$ $L^{t}(\mu)$ if $1 \leq t<2$ while $P^{t}(\mu)$ is a space of analytic functions if $t>2$. For our second example $\sigma$ we add a weighted area measure to $\mu$. The set of bpes for $P^{3}(\sigma)$ is $U$, but the set of bpes for $P^{1}(\sigma)$ is $U \backslash[0,1)$.

\section{BACKGROUND AND SEIP'S THEOREMS}

Define for each $n>0$ the weighted Bergman space $A^{-n, 2}$ to be the Banach space of functions in $L^{2}\left(\left(1-|z|^{2}\right)^{2 n-1} d A\right)$ that are analytic in $U$. Observe that there exist positive constants $c_{j}$ (depending on $n$ ) such that for $f(z)=\sum_{j} a_{j} z^{j}$ in $A^{-n, 2}$

$$
\|f\|^{2}=(1 / \pi) \iint|f|^{2}\left(1-r^{2}\right)^{2 n-1} r d r d \theta=\sum_{j} c_{j}\left|a_{j}\right|^{2} .
$$

Thus, the partial sums of the Taylor series for $f$ converge to $f$ in norm. Consequently, $A^{-n, 2}=P^{2}\left(\left(1-|z|^{2}\right)^{2 n-1} d A\right)$.

Now we follow Seip [7]. Let

$$
\rho(z, w)=\left|\frac{z-w}{1-\bar{z} w}\right|
$$

which is the pseudohyperbolic distance function on $U$. We say that a sequence $\Gamma=\left\{z_{j}\right\}$ is uniformly discrete (or separated) if

$$
\inf _{j \neq k} \rho\left(z_{j}, z_{k}\right)>0 \text {. }
$$

For a uniformly discrete set $\left\{z_{j}\right\}$ and $\frac{1}{2}<r<1$ let

$$
D(\Gamma, r)=\frac{\sum \log \frac{1}{\left|z_{j}\right|}}{\log \frac{1}{1-r}}
$$

where the sum is taken over all $j$ with $\frac{1}{2}<\left|z_{j}\right|<r$. For each $z$ in $U$ we form a new sequence

$$
\Gamma_{z}=\left\{\frac{z_{j}-z}{1-\bar{z} z_{j}}\right\} \text {. }
$$

The lower and upper uniform densities of $\Gamma$ are defined, respectively, as

$$
D^{-}(\Gamma)=\liminf _{r \rightarrow 1} \inf _{z \in U} D\left(\Gamma_{z}, r\right)
$$

and

$$
D^{+}(\Gamma)=\limsup _{r \rightarrow 1} \sup _{z \in U} D\left(\Gamma_{z}, r\right)
$$


The key example of a sequence is the following sequence from Seip [7]. For $a>1, b>0$, let $\Gamma$ denote the image of $\left\{a^{j}(b k+i)\right\}_{j, k \in Z}$ under the Cayley transform of the upper half-plane to $U$. Then

$$
D^{-}(\Gamma)=D^{+}(\Gamma)=\frac{2 \pi}{b \log a} .
$$

The following relationship between atomic measures and area measure is an immediate consequence of [7, Equation (2)]. Let $\left\{z_{j}\right\}$ be a uniformly discrete sequence in $U$, and let $\delta=\inf _{j \neq k} \rho\left(z_{j}, z_{k}\right)$. Then for $f$ analytic in $U$

$$
\sum\left(1-\left|z_{j}\right|^{2}\right)^{s}\left|f\left(z_{j}\right)\right|^{2} \leq C(\delta) \int\left(1-|z|^{2}\right)^{s-2}|f(z)|^{2} d A(z)
$$

whenever $s>0$ (both sides may be infinite).

A sequence $\left\{z_{j}\right\}$ of distinct points in $U$ is a set of sampling for $A^{-n, 2}$ if there exist positive constants $K_{1}$ and $K_{2}$ such that

$$
\begin{aligned}
K_{1} \int|f|^{2}\left(1-|z|^{2}\right)^{2 n-1} d A(z) & \leq \sum\left|f\left(z_{j}\right)\right|^{2}\left(1-\left|z_{j}\right|^{2}\right)^{2 n+1} \\
& \leq K_{2} \int|f|^{2}\left(1-|z|^{2}\right)^{2 n-1} d A(z)
\end{aligned}
$$

for every $f$ in $A^{-n, 2}$. The sequence $\left\{z_{j}\right\}$ is a set of interpolation for $A^{-n, 2}$ if for every sequence $\left\{a_{j}\right\}$ for which $\sum\left(1-\left|z_{j}\right|^{2}\right)^{2 n+1}\left|a_{j}\right|^{2}<\infty$ there exists a function $f$ in $A^{-n, 2}$ such that $f\left(z_{j}\right)=a_{j}$ for all $j$.

We now state Seip's theorems for weighted Bergman spaces [7].

Theorem 2.1. A sequence $\Gamma$ of distinct points in $U$ is a set of sampling for $A^{-n, 2}$ if and only if it can be expressed as a finite union of uniformly discrete sets and it contains a uniformly discrete subsequence $\Gamma^{\prime}$ for which $D^{-}\left(\Gamma^{\prime}\right)>n$.

Theorem 2.2. A sequence $\Gamma$ of distinct points in $U$ is a set of interpolation for $A^{-n, 2}$ if and only if $\Gamma$ is uniformly discrete and $D^{+}(\Gamma)<n$.

\section{EXAMPLES}

Let $n>0$, and let $\Gamma=\left\{z_{j}\right\}$ be a uniformly discrete sequence with $D^{+}(\Gamma)=$ $D^{-}(\Gamma)=n$. Let $d=\inf _{j \neq k} \rho\left(z_{j}, z_{k}\right)$. For $z$ in $U$ let $\delta_{z}$ denote the measure of point mass at $z$. Let $\alpha$ be the sigma-finite measure $\sum \delta_{z_{j}}$.

Let $\mu$ be the measure with $d \mu=\left(1-|z|^{2}\right)^{2 n+1} d \alpha$. Using (1) with $f \equiv 1$, we see that

$$
\int\left(1-|z|^{2}\right)^{2 n+1} d \alpha \leq C(d) \int\left(1-|z|^{2}\right)^{2 n-1} d A(z)<\infty .
$$

Thus, the measure $\mu$ is finite. This argument also applies to each atomic measure introduced in the proof of the following theorem.

Theorem 3.1. If $1 \leq t<2$, then $P^{t}(\mu)=L^{t}(\mu)$. If $t>2$, then $P^{t}(\mu) \neq L^{t}(\mu)$. Proof. First consider the case where $1 \leq t<2$. Let $s=t / 2$. Choose $m>n$ such that $s m<n$, and let $\varepsilon=(m-n) /(1-s)$. Let $\nu$ be the measure with $d \nu=\left(1-|z|^{2}\right)^{2 m+1-2 \varepsilon} d \alpha$.

Because $\varepsilon<m$, the measure $\nu$ is finite. Also the equality

$$
2 n+1=2 m+1-2 \varepsilon+2 \varepsilon s
$$


implies that

$$
d \mu=\left(1-|z|^{2}\right)^{\varepsilon t} d \nu .
$$

Let $\tau$ be the measure with $d \tau=\left(1-|z|^{2}\right)^{2 \varepsilon} d \nu$.

Now let $q$ be the positive number with $(1 / q)+s=1$. Let $p$ be a polynomial. By Holder's inequality

$$
\begin{aligned}
\int|p|^{t} d \mu & =\int|p|^{t}\left(1-|z|^{2}\right)^{\varepsilon t} d \nu \\
& \leq\left(\int|p|^{2}\left(1-|z|^{2}\right)^{2 \varepsilon} d \nu\right)^{s}\|\nu\|^{1 / q} .
\end{aligned}
$$

Hence there is a constant $C$, independent of the polynomial $p$, such that

$$
\|p\|_{L^{t}(\mu)} \leq C\|p\|_{L^{2}(\tau)} \text {. }
$$

Because $m>D^{+}(\Gamma)$, it follows from Theorem 2.2 that $\Gamma$ is interpolating for $A^{-m, 2}$. Using (1) and noting that $d \tau=\left(1-|z|^{2}\right)^{2 m+1} d \alpha$, we see that the characteristic function of each singleton is in $P^{2}(\tau)$. It now follows from (2) that each such characteristic function is in $P^{t}(\mu)$. Thus $P^{t}(\mu)=L^{t}(\mu)$.

Next consider the case where $t>2$. Again let $s=t / 2$. Choose $m$ such that $0<m<n$ and $n<m s$. Define $\varepsilon$ and $\nu$ symbolically the same as in the previous case. Note that we again have $0<\varepsilon<m$ and $d \mu=\left(1-|z|^{2}\right)^{\varepsilon t} d \nu$. Let $\tau$ be the measure with $d \tau=\left(1-|z|^{2}\right)^{2 m-1} d A$.

Now let $q$ be the positive number with $(1 / q)+(2 / t)=1$. Let $p$ be a polynomial. Because $m<D^{-}(\Gamma)$, it follows from Theorem 2.1 that $\Gamma$ is a set of sampling for $A^{-m, 2}$. Thus there exists a constant $C$ such that

$$
\begin{aligned}
\int|p|^{2}\left(1-|z|^{2}\right)^{2 m-1} d A & \leq C \int|p|^{2}\left(1-|z|^{2}\right)^{2 m+1} d \alpha \\
& =C \int|p|^{2}\left(1-|z|^{2}\right)^{2 \varepsilon} d \nu \\
& \leq C \int\left(|p|^{t}\left(1-|z|^{2}\right)^{\varepsilon} d \nu\right)^{2 / t}\|\nu\|^{1 / q} .
\end{aligned}
$$

It now follows that there exists a positive constant $K$ such that

$$
\|p\|_{L^{2}(\tau)} \leq K\|p\|_{L^{t}(\mu)} .
$$

Observing that each point in $U$ is a bpe for $P^{2}(\tau)$, we see that $U$ equals the set of bpes for $P^{t}(\mu)$.

Remark. In the case where $P^{t}(\mu)=L^{t}(\mu)$ the set of bpes for $P^{t}(\mu)$ equals the set of atoms of $\mu$. It is obvious that each atom gives rise to a bpe, so it suffices to consider a point $\lambda$ that is not an atom. Since $(z-\lambda) L^{t}(\mu)$ is dense in $L^{t}(\mu)$, it follows that the polynomials that vanish at $\lambda$ are dense in $L^{t}(\mu)$ also. In particular, the constant function one is in the closure of the set of polynomials that vanish at $\lambda$. But the constant function one takes on the value one at each bpe, so $\lambda$ cannot be a bpe.

Theorem 3.2. There exists a measure $\sigma$ such that the set of bpes for $P^{3}(\sigma)$ equals $U$ and the set of bpes for $P^{1}(\sigma)$ equals $U \backslash[0,1)$.

Proof. Let $n, \Gamma$, and $\mu$ be as indicated at the start of this section. Applying a Mobius transformation to $\Gamma$, if necessary, we may assume that $\Gamma$ does not meet the interval $[0,1)$. 
Now let $t=1$, and choose $m$ and $\tau$ as in the first part of the proof of Theorem 3.1. Recall that $\Gamma$ is an interpolating sequence for $A^{-m, 2}$. Thus, there exists a nonconstant function $f$ in $A^{-m, 2}$ that vanishes on $\Gamma$. Let $u(z)=\left(1-|z|^{2}\right)^{2 m-1}$, so $P^{2}(u d A)=A^{-m, 2}$. Using (1), we see that each sequence of polynomials converging to $f$ in $P^{2}(u d A)$ also converges to $f$ in $P^{2}(\tau)$. Now using (2), we see that each such sequence also converges to $f$ in $P^{1}(\mu)$. Thus, $f$ belongs to $P^{1}(d \mu+u d A)$.

By a method of $\mathrm{W}$. Hastings [4; 3, p. 83], there is a weight function $w$ defined on $U \backslash[0,1)$ such that (a branch of) $z^{1 / 2}$ belongs to $P^{1}(w|f| u d A)$. Furthermore, we may assume that $0<w \leq 1$ and $w$ is bounded below on each compact subset of $U \backslash[0,1)$.

Let $\sigma$ be the measure with $d \sigma=d \mu+w u d A$. Since $u$ and $w$ are bounded below on each compact subset of $U \backslash[0,1)$, it follows that each point in $U \backslash[0,1)$ is a bpe for $P^{1}(\sigma)$. Recalling that $\Gamma$ does not meet $[0,1)$, we may conclude that $P^{1}(\sigma)$ contains no nontrivial $L^{1}$-summand. By [8] it follows that the set of bpes is open and that each function in $P^{1}(\sigma)$ extends to be analytic on the set of bpes.

The function $f$ above is in $P^{1}(\sigma)$ because $w \leq 1$. It follows from the defining property of $w$ that $z^{1 / 2} f$ belongs to $P^{1}(\bar{\sigma})$. But $z^{1 / 2} f$ cannot be extended to be analytic in any region containing a point on $[0,1)$. Thus, the set of bpes for $P^{1}(\sigma)$ equals $U \backslash[0,1)$.

Since the set of bpes for $P^{3}(\mu)$ equals $U$, the same conclusion holds for $P^{3}(\sigma)$.

\section{REFERENCES}

1. J. Akeroyd, Polynomial approximation in the mean with respect to harmonic measure on crescents, Trans. Amer. Math. Soc. 303 (1987), 193-199.

2. J. Brennan, Invariant subspaces and weighted polynomial approximation, Ark. Mat. 11 (1973), 167-189.

3. J. B. Conway, The theory of subnormal operators, Math. Surveys Monographs, vol. 36, Amer. Math. Soc., Providence, RI, 1991.

4. W. W. Hastings, A construction in Hilbert spaces of analytic functions, Proc. Amer. Math. Soc. 74 (1979), 295-298.

5. S. N. Mergeljan, On the completeness of systems of analytic functions, Amer. Math. Soc. Transl. Ser. 2, vol. 19, Amer. Math. Soc., Providence, RI, 1962, pp. 109-166; Uspekhi Mat. Nauk 8 (1953), 3-63.

6. K. Seip, Regular sets of sampling and interpolation for weighted Bergman spaces, Proc. Amer. Math. Soc. 117 (1993), 213-220.

7. __ Beurling type density theorems in the unit disk, Invent. Math. 113 (1993), 21-39.

8. J. E. Thomson, Approximation in the mean by polynomials, Ann. of Math. (2) 133 (1991), 477-507.

Department of Mathematics, Virginia Tech, Blacksburg, Virginia 24061-0123

E-mail address: thomson@math.vt.edu 